\title{
PREFACE
}

\section{Attention Deficit Hyperactivity Disorder}

$\mathrm{B}_{\text {ack by popular demand! It is a pleasure }}$ to have Guest Editor Jack Damico back hosting an issue updating information for clinicians and researchers working with children who have attention deficit hyperactivity disorder (ADHD). Part of the commitment of Seminars is to offer such periodic updates on topics of continuing interest for speech-language pathologists. Since our 1996 issue, readers have written to request that we provide a follow-up volume, and we are pleased to do so now.

There is no question that children and teenagers with ADHD form a substantial number of individuals on the caseloads of speech-language pathologists in the United States as well as abroad, as contributors Clegg and Hartshorne note in their article. In recognition of the relevance that ADHD has for effective academic and social uses of language, the American Speech-Language-Hearing Association has long taken the position that speech-language pathologists and audiologists "play vital roles in the assessment, diagnosis and treatment of persons of all ages with attention deficit hyperactivity disorder (ADHD)." ${ }^{1}$ Well-delineated roles include being part of the team that diagnoses the condition, serving as primary service providers for people who have ADHD and communication disorders and consulting with families and other professionals to help maximize educational, family, and social performance.

As contributor Tetnowski notes, a significant number of children with ADHD appear to have concurrent primary language-learning or central auditory processing disorders, although separating the impact of ADHD on the usual measures used to diagnose these conditions can be quite challenging. The hyperactivity that classically accompanies ADHD and is often one of its first presenting symptoms can begin to have an impact on educational performance as early as the preschool years, as Armstrong and Nettleton note in their article. However, it is not always clear that ADHD is in the actions of the child, rather than in the expectations of the teachers and speech-language pathologists, as Pierce and Reid observe in their analysis of the challenges faced when making a diagnosis of ADHD in children from culturally diverse backgrounds. Because a primary treatment option for children with ADHD is pharmaceutical, misdiagnosis can have significant health, behavioral, and financial ramifications on the lives of the child and his or her family.

Contributors Westby and Watson and Nelson and Hawley present updated conceptualizations of the mechanisms that govern the impact of ADHD on language, reading, and communication, all of which have important ramifications for understanding the child and addressing core deficits that produce significant functional outcomes. Readers often request a practitioner's view of how to translate basic research into everyday clinical practice; Dunaway's contribution is an excellent illustration of how to implement evidence-based practice in working with children who have ADHD, given what we know and have yet to learn about this complex disorder.

Again, I thank Professor Damico for shepherding this important collection to publication. As always, the Editors of Seminars welcome suggestions for additional "by request" topics from its readers. You may contact me directly with suggested topics or other comments regarding our pediatric-focus series at nratner@hesp.umd.edu 


\section{REFERENCE}

1. American Speech-Language-Hearing Association. Position statement: roles of audiologists and speechlanguage pathologists working with persons with attention deficit hyperactivity disorder: position statement and technical report. ASHA 1996; 39(Suppl 17):273-300

Nan Bernstein Ratner, Ed.D. Co-Editor in Chief ${ }^{1}$ 